\title{
Optimal Kinematic Design of a Haptic Pen
}

\author{
L.J. Stocco, member, IEEE, S.E. Salcudean, member, IEEE, and F. Sassani
}

\begin{abstract}
This paper investigates the performance demands of a haptic interface and shows how this information can be used to design a suitable mechanism. A design procedure, previously developed by the authors, that consists of a global isotropy index and a discrete optimization algorithm, allows one to compare a range of geometric variables, actuator scale factors and even different robot devices for optimum performance. The approach is used to compare the performance of three 6-DOF robots including two well-known parallel platform robots and a novel hybrid robot called the Twin-Pantograph in terms of their semidextrous workspaces and static force capabilities. Since the TwinPantograph yields the best results, its design is refined to address practical constraints and it is implemented as a haptic pen. The performance of the haptic pen is then measured to evaluate the effectiveness of the design procedure.
\end{abstract}

\section{INTRODUCTION}

Haptic interfaces present a difficult mechanical design problem. It is just as important for a haptic device to be light and backdriveable as it is for it to be stiff and unyielding. Since maximum stiffness and compliance are compromised with almost every design variable, the primary challenge is to broaden the range of achievable impedances [5]. Robot configurations, geometric parameters, transmission ratios, external dampers and actuator redundancy have all been proposed as a means of widening this range.

Lawrence and Chapel [15] suggest that there is a limit to the useful impedance range of a haptic device. It is upper bounded by the stiffness required to counteract a reasonable maximum hand force and lower bounded by the largest impedance that is too small for a human to detect. Any impedances outside of this range are beyond the scope of human perception and are not relevant to the performance of a haptic device.

There have been many proposals on how to widen the impedance range of a haptic interface. Lawrence and Chapel [15] propose lowering effective mass while Hayward et al. [9], Massie and Salisbury [20] and Ma and Angeles [19] make an argument for reducing variations in mass. Kurtz and Hayward [14] and Zanganeh and Angeles [29] argue the merits of an isotropic Jacobian while Colgate and Schenkel [5] suggest increasing stability through physical damping.

These, and other design philosophies have led to a wide variety of physical devices. Iwata [10] built a 9-DOF Compact Master which combines a 6-DOF parallel positioning device in series with three 1-DOF finger actuators. Iwata [11] also used a pair of commercial 3-DOF hybrid manipulators to actuate each end of a 6-DOF haptic pen. In [4], the 6-DOF hybrid SMARTee interface uses a parallel combination of three 2-DOF serial linkages. In [18] a 6-DOF joystick is described which contains three pantograph linkages and Yokoi et al. [28] present a 6-DOF serial hand controller with three prismatic actuators for translation and three rotary actuators for orientation. A 5-DOF (3 translation, 2 rotation) haptic stylus is described in [16] which uses 5 linear actuators in parallel. A 4-DOF ( 3 translation, 1 rotation) hybrid device using rotary actuators is presented in [13] while a 4-DOF Manipulandum that combines a 3-DOF planar parallel robot in series with a linear vertical motion stage is described in [21]. In [20], the hybrid PHANToM has three active translational degrees of freedom and three passive rotational degrees of freedom. A 2DOF planar pantograph is optimized in [9] and the 2-DOF linear actuated Magic Mouse is presented in [12]. Finally, Vertut [26] presents a historical survey of earlier hand controllers, articulated arms and exoskeletons.

In this paper, a further attempt is made to identify the mechanical properties that lead to a wider impedance range and to design a device that bears them. In Section II, a model of a human hand interacting with a haptic interface is used to motivate the choice of static force capabilities as the kinematic design criteria. Section III describes two biomechanics studies that are conducted to obtain force and velocity values to guide the kinematic design and sensor selection processes. In Section IV, a design procedure is summarized which was previously developed by the authors in [24] and [25]. It selects the geometric parameters and actuator scale factors that best satisfy a direction dependent performance criteria inside a pre-defined operational workspace. It includes a normalized Global Isotropy Index and a discrete minimax optimization algorithm called the culling algorithm. In Section V, the proposed specifications and design procedure are used to compare three six-degree-of-freedom robots including the Stewart platform [8], the Inoue platform [6] and a novel hybrid robot called the Twin-Pantograph for workspace size and static force capabilities. Since the TwinPantograph turns in the best results, it is implemented as a haptic pen. Section VI describes the details of its physical design, the control algorithm used to simulate two virtual environments and reports some performance measurements. Section VII summarizes the results and presents conclusions. 


\section{EVALUATING HAPTIC PERFORMANCE}

Many design specifications are relevant to the performance of a haptic interface. They include workspace size, position bandwidth, force magnitude, force bandwidth, velocity, acceleration, effective mass, accuracy, and so on. A survey of proposed values for many of these design specifications can be found in [23]. Most of these values are derived from the inherent capabilities of the human hand since this is the environment of a haptic interface.

A human hand is comprised of a skeletal structure which presents a position dependent impedance $Z_{s}$ and muscles which can either contract or stiffen to present a force $f_{h}$ and/or an impedance $Z_{m}$ to its environment. In Figure 1, the total hand impedance $Z_{h}$ is the combination of voluntary muscular impedance $Z_{m}$ and involuntary skeletal impedance $Z_{s}$. Its combined behaviour is adapted by the nervous system to simulate a force source when interacting with a stiff environment (e.g. an open-circuit), a velocity source when navigating a compliant environment (e.g. a short-circuit) and an impedance when restraining a non-passive environment (e.g. an active source).

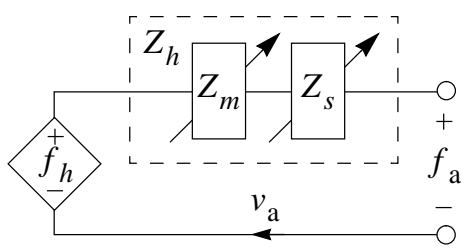

Fig. 1. Human Hand Model

A haptic interface displays tactile information by presenting an impedance to the human hand. Of course, impedance must be varied in an intelligent fashion so the device includes active sources, but to ensure stability, these sources can only be used to dissipate energy and must not supply any. Two types of devices can be used to implement an intelligent variable impedance, an impedance device and an admittance device. An impedance device is easily backdriveable in its passive state and typically uses direct drive or moderately geared actuators to adjust its effective impedance by applying a force based on position and velocity. An admittance device is the dual of an impedance device. It is not backdriveable in its passive state and typically uses highly geared actuators to adjusts its effective impedance by inducing motion based on applied force.

An impedance device senses velocity $v_{a}$, position $s^{-1} v_{a}$ and possibly acceleration $s v_{a}$ and responds with a force $f_{a}$ comprised of an active force $f_{r}$ from the motors and a passive force from the mechanical impedance $Z_{r}(s)$ of the device where $s$ is the Laplace transform variable. The effective impedance experienced by the hand $Z_{e}(s)$ (1) may contain effective mass $M_{e}$, damping $B_{e}$ and stiffness $K_{e}$ terms (3) which are contributed in part by the physical mass $M_{r}$ and damping $B_{r}$ of the robot mechanism (2).

$$
\begin{gathered}
f_{a}=Z_{r}(s) v_{a}+f_{r}=Z_{e}(s) v_{a} \\
Z_{r}(s)=M_{r} s+B_{r} \\
Z_{e}(s)=M_{e} s+B_{e}+K_{e} s^{-1}
\end{gathered}
$$

An admittance device senses applied force $f_{a}$ and responds by inducing a velocity $v_{a}$ which is comprised of both active velocity $v_{r}$ from the motors and passive velocity due to the robot's nonzero mechanical admittance $Y_{r}(s)$. The total effective admittance experienced by the hand $Y_{e}(s)$ is shown in (4).

$$
v_{a}=Y_{r}(s) f_{a}+v_{r}=Y_{e}(s) f_{a}
$$

Combining the human hand model with the haptic device models results in the haptic systems shown in Figure 2 where $Z_{c}(s)$ in (5) and $Y_{c}(s)$ in (6) are the impedance and admittance simulated through active control.

$$
\begin{aligned}
& Z_{c}(s)=v_{r} / f_{a} \\
& Y_{c}(s)=f_{r} / v_{a}
\end{aligned}
$$

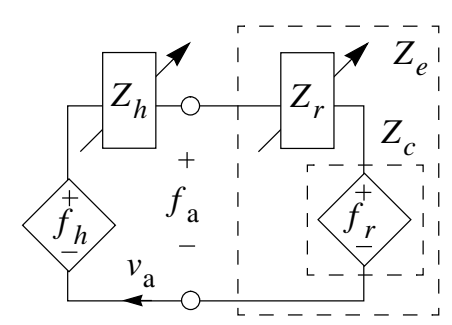

Impedance System

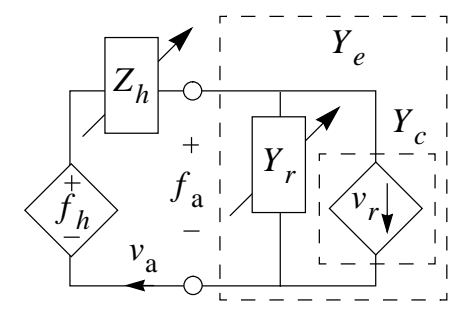

Admittance System
Fig. 2. Haptic Systems

The high inertia and friction that is inherent in a highly geared admittance device inhibits high frequency transitions (i.e. switching between free motion and hard contact) and is difficult to actively compensate resulting in low transparency and sluggish free motion that can be tiring during prolonged use. Therefore, we are more interested here in design issues related to impedance devices.

As discussed by Colgate and Schenkel [5], it is difficult to actively compensate for the physical dynamics of a mechanical system without compromising stability. Therefore, the minimum effective impedance of a haptic device is improved by a reduction in mechanical impedance. Salcudean and Vlaar [22] also show that even when high static forces are not available, high stiffnesses can be simulated by inducing high acceleration. Therefore, it follows that both high and low impedance emulation are improved and the impedance range is widened when mechanical impedance is reduced. Reducing mechanical impedance can be done in a number of ways that do not necessarily involve kinematic design and the mass matrix. It may in fact be sufficient to use a parallel device, light weight materials, low friction joints and counterbalances so that kinematic design can be focussed on other criteria such as force. 
From Figure 2, it is apparent that if $Z_{r}$ is sufficiently small, an impedance device's performance is dictated by its force capabilities. Since actuators can always be scaled to meet any force magnitude requirements, it does not make much sense to maximize force through kinematic design. It is more important for the force capabilities to match those of the human hand along different directions of motion and for those capabilities to be consistent throughout the workspace. Then, actuators are fully utilized regardless of direction. This allows smaller actuators to be used which results in lower rotor inertia, consistently stiff virtual environments, improved compactness and lower cost.

A similar argument can be made for measurement accuracy. Sensor resolution can always be increased to meet minimum standards but a device that is kinematically matched to its environment will tolerate coarser sensor resolutions and exhibit greater consistency with a smaller price tag and footprint.

For the impedance device designed here, the geometric and actuator design parameters are selected so that the device's force capabilities match those of the human hand as consistently as possible throughout the workspace. The velocity capabilities of the human hand are considered afterward to select sensor resolutions and the mechanical impedance is minimized by using a device which has most of its actuators in the base and components with low mass and friction.

\section{PERFORMANCE SPECIFICATIONS}

For tangible static force requirements, the maximum force/torque capabilities of the human hand are needed. While existing data on maximum sustainable force is available in the human-factors and haptic interfaces literature, it is not easily applied to haptic interface design. For example, in [1], [2] and [27], maximum forces and torques are measured using a hammer grip. This type of grip enables larger forces but less precision than the pencil grip preferred for a desktop haptic device. Other human force/torque measurements can be found in [3] and [21] but they do not distinguish between capabilities in different directions. To obtain this information, a biomechanics experiment was conducted.

The experiment involved 20 participants ( 12 males, 8 females) ranging between 20-60 years and $45-90 \mathrm{Kg}$. Participants included right and left handed people from a variety of disciplines including students, professors, engineers, secretaries and athletes. The test apparatus was an aluminum rod with the same diameter as a standard wooden pencil $(0.63 \mathrm{~cm})$ fixed at each end to a 6 axis force/torque sensor. The rod was oriented vertically, covered with athletic tape to improve grip and mounted in front of an armrest to minimize the forces contributed by the arm and shoulder. A photograph of the test apparatus is shown in Figure 3.

participants grasped the rod using a pencil grip and were instructed to push, pull and twist the rod in all six directions using a consistent amount of effort. They alternately applied

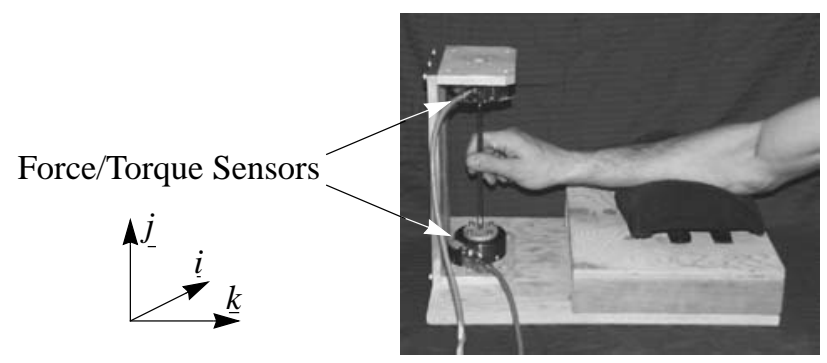

Fig. 3. Force/Torque Test Apparatus

static (push and hold) force/torque in both the positive and negative directions and dynamic (back and forth) force/torque along each of the 6 axes. A typical data set obtained from the force/torque sensors is shown in Figure 4 where $F$ and $M$ are forces and moments about axes $\underline{i}, \underline{j}$ and $\underline{k}$.

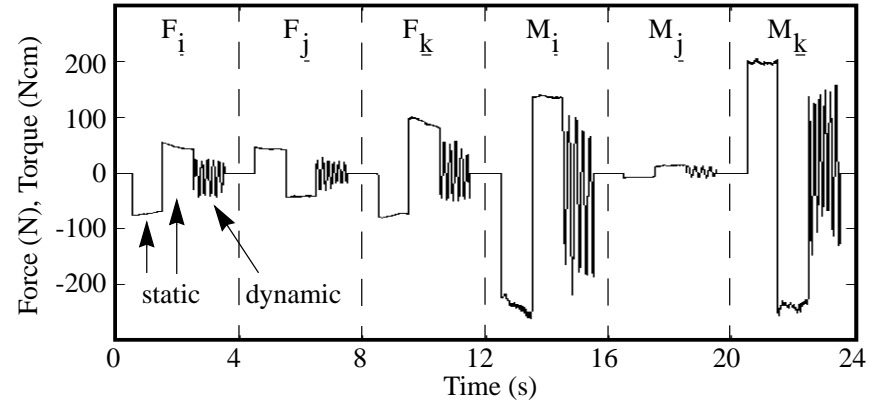

Fig. 4. Typical Force/Torque Data

The task was performed twice by each participant, once with high exertion and again with medium exertion where the amount of effort for "high" and "medium" exertion was subjectively determined by each participant. Forces and torques were averaged by calculating the RMS values of a one second sampling period. The highest and lowest $10 \%$ of the RMS values were removed to account for experimental error and the remaining 80\% were averaged. The mean (plotted point) and standard deviation (error bar) for both the static and dynamic trials are shown in Figure 5.
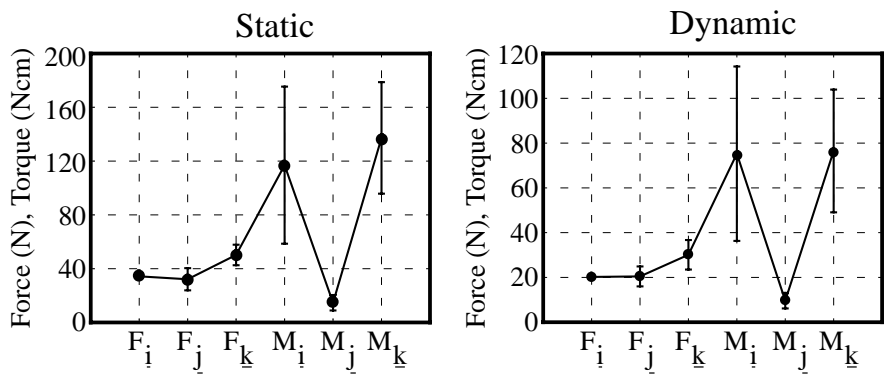

Fig. 5. Force/Torque Test Values

Since the results of the static and dynamic tests differ by less than $10 \%$ and have reasonable standard deviations, the data is assumed to be reliable. The static and dynamic values were 
averaged and normalized with respect to $F_{i}$ (force about the $i$ axis) to obtain the relative values shown in Table I.

TABLE I

RELATIVE FORCE/TORQUE CAPABILITIES OF THE HUMAN HAND

\begin{tabular}{c|c|c|c}
\cline { 2 - 4 } & $\underline{i}$ axis & $j$ axis & $\underline{k}$ axis \\
\hline Force $/ F_{\underline{i}}$ & 1.0 & 1.0 & 1.5 \\
\hline Torque $/ F_{\underline{i}}$ & $3.6 \mathrm{~cm}$ & $0.5 \mathrm{~cm}$ & $3.9 \mathrm{~cm}$
\end{tabular}

To obtain tangible position/velocity sensing requirements, the maximum velocity capabilities of the human hand were also identified. This involved a biomechanics experiment that measured the maximum achievable frequencies of periodic linear and angular motions of different amplitudes. Each experiment involved 10 participants ( 5 males, 5 females) from a distribution of ages, weights and professions similar to that in the force/torque experiment. The test apparatus for the translational experiment was a pencil and paper with six rectangles drawn on it (see Figure 6). The rectangles were $6 \mathrm{~cm}$ high and ranged in width from $0.15 \mathrm{~cm}$ to $16 \mathrm{~cm}$. Participants were asked to shade in the rectangles as fast as possible while their hand trajectory was recorded by a position tracking device (Bird ${ }^{\mathrm{TM}}$ magnetic sensor). The test apparatus for the rotational experiment was a low friction potentiometer connected to a handle through a universal joint (see Figure 6). The trajectory was recorded and displayed in real time on a CRT with visual upper and lower amplitude bounds. Participants were asked to oscillate between the bounds as quickly as possible and were free to orient the test apparatus to maximize comfort and speed. Note that although the rotational test apparatus has a flat handle, it is held with a grip that is very similar to a pencil grip.
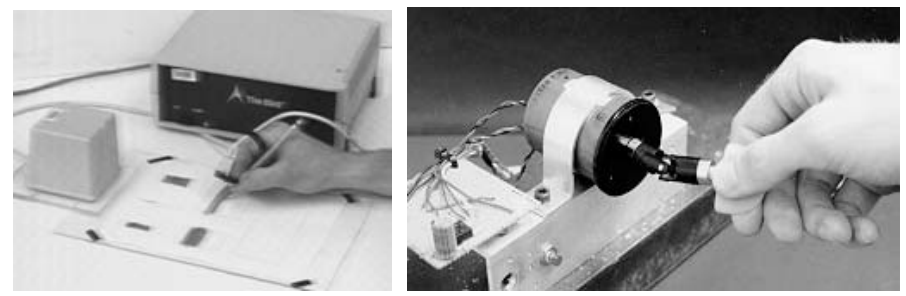

Fig. 6. Velocity Test Apparatus

A typical data set obtained from one trial of the rotation experiment is shown in Figure 7. Translational data looks similar and is not shown.

The trials were performed three times by each participant. The trajectories were analyzed to obtain the dominant frequency component and the results of all trials were averaged. Maximum velocity was computed by assuming a sinusoidal trajectory as shown in equations (7) through (9) and is plotted in Figure 8. These plots provide the maximum speed that the human hand can achieve inside a workspace of up to $16 \mathrm{~cm}$ in translation and $180^{\circ}$ in rotation.

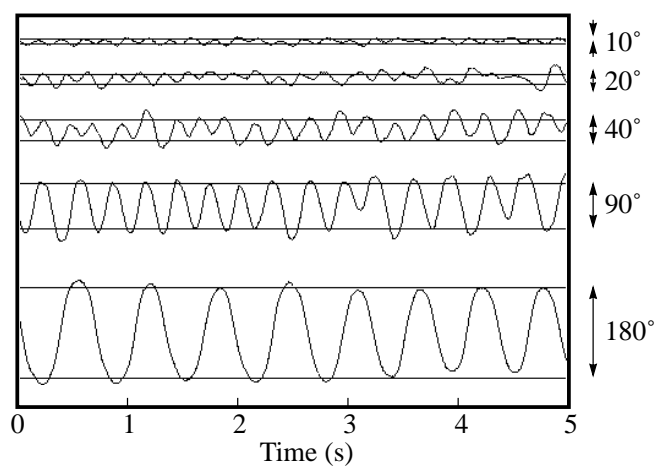

Fig. 7. Typical Trajectories From Angular Velocity Test

$$
\begin{gathered}
x=A \sin (\omega t) \\
\dot{x}=A \omega \cos (\omega t) \\
\|\dot{x}\|=A \omega
\end{gathered}
$$
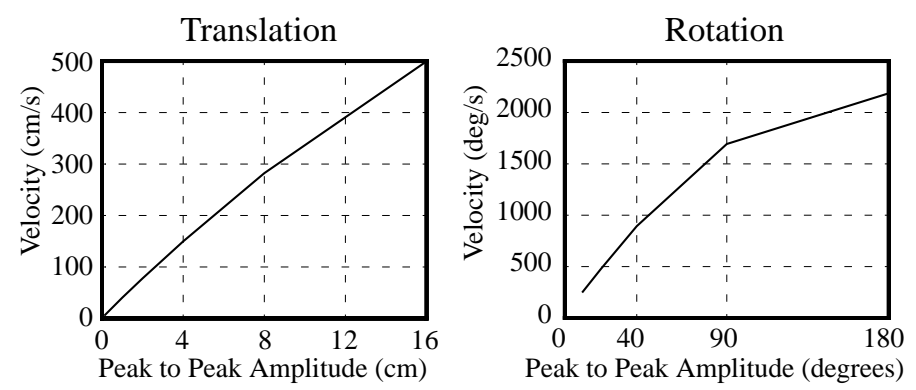

Fig. 8. Velocity Test Results

\section{DESIGN PROCEDURE}

The goal of the design procedure is to solve the optimization problem in (10) by finding the robot design parameter $p^{*}$ that maximizes the condition index $F(p)$ where $p$ is a design parameter belonging to a set of candidates $P$. The condition index $F(p)$ should take into account the intended use of the robot and variations in performance at different workspace positions.

$$
p^{*}=\underset{p \in P}{\arg \max }(F(p))
$$

For the haptic pen designed here, the Global Isotropy Index, proposed in [24], is used as the condition index $F(p)$. The matrix normalizing technique described in [25] is applied to the GII to obtain the workspace-inclusive, unitless, task-dependent performance measure shown in equation (11) where $\sigma$ and $\tilde{\sigma}$ are the minimum and maximum singular values of the Jacobian $J(p, x)$ which is computed for a design parameter $p$ at a position $x$ in the workspace $W$ and is scaled by matrices $S_{J}(12)$ and $S_{T}(13)$ which are diagonal matrices containing the maximum torque capabilities $\tau_{1} \ldots \tau_{n}$ of each actuator (for a robot with $n$ actuators) and the maximum required end-effector forces $F$ and moments $M$ about axes $\underline{i}, \underline{j}$ and $\underline{k}$. 


$$
\begin{gathered}
G I I(p)=\min _{x_{0}, x_{1} \in W} \frac{\sigma\left(S_{J} J\left(p, x_{0}\right) S_{T}^{-1}\right)}{\tilde{\sigma}\left(S_{J} J\left(p, x_{1}\right) S_{T}^{-1}\right)} \\
S_{J}=\operatorname{Diag}\left(1 / \tau_{1}\left[\begin{array}{llll}
\tau_{1} & \tau_{2} & \ldots & \tau_{n}
\end{array}\right]\right) \\
S_{T}=\operatorname{Diag}\left(1 / F_{i}\left[F_{i} F_{j} F_{k} M_{i} M_{j} M_{k}\right]\right)
\end{gathered}
$$

By fixing the elements of $S_{T}$ to match the requirements of the application, the GII is customized to reflect how well a particular design satisfies those requirements. By choosing the elements of $S_{J}$ as free design parameters, the optimum robot geometry and actuator sizes are identified simultaneously. This has been shown in [25] to significantly improve robot performance, particularly with serial mechanisms.

To solve the minimax problem from combining equations (10) and (11), the culling algorithm [24] is used. The culling algorithm is a discrete optimization algorithm that is specifically designed to handle GII and minimax problems. It guarantees the same result as a global search but arrives at it orders of magnitude faster. It can be used with any performance function, is insensitive to initial conditions and places no theoretical limit on the number of free variables. Search ranges and resolutions are limited only by memory and time constraints. It has been shown in [24] to be extremely efficient at solving robot design problems.

Since the culling algorithm is a discrete optimization algorithm, the workspace must be discretized. This is fine for translations but non-uniform sample spacing [17] and an unsuitable workspace boundary (see Figure 9a) result if Euler angles are used to compute rotations. An axis-angle representation $R=\left(\theta_{0}, \theta_{1}, \theta_{2}\right)$ (see Figure 9) leads to a more suitable workspace boundary by bounding $\theta_{0}$ and $\theta_{1}$ while leaving $\theta_{2}$ unconstrained $\left(0 \leq \theta_{2}<2 \pi\right)$.
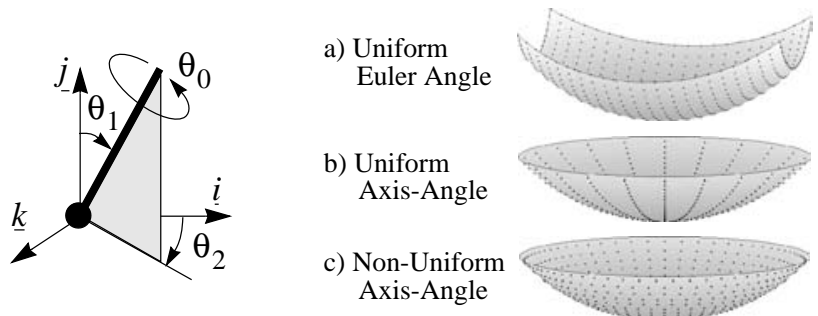

Fig. 9. Comparison of Angular Workspaces Using 3

Unfortunately, sample spacing is still non-uniform if $\theta_{0}, \theta_{1}$ and $\theta_{2}$ are discretized uniformly (see Figure $9 \mathrm{~b}$ ). To homogenize sample spacing, $\theta_{1}$ is discretized uniformly (i.e. constant $\Delta \theta_{1}$ ) but the sample spacing of $\theta_{2}\left(\Delta \theta_{2}\right)$ is computed individually for each $\theta_{1}$ value from equation (14).

$$
\Delta \theta_{2, n}=\frac{\Delta \theta_{1}}{\sin \left(n \Delta \theta_{1}\right)} ; n \in\{1,2,3, \ldots, N\}
$$

The three discretization methods are compared in Figure 9 using a bound of $45^{\circ}$ for $\theta_{l}$. For each sample, a point is plotted on the solid angle that defines the boundary of the angular workspace. Notice the favourable workspace boundary and uniform distribution resulting from non-uniform Axis-Angle sampling.

To ensure an equitable distribution between the total number of translational $\left(k_{t}\right)$ and rotational $\left(k_{r}\right)$ samples, their geometric means are made equal by computing $k_{r}$ from (15) where $k_{i}, k_{j}$ and $k_{k}$ are the number of samples along each translational axis and $d_{t}$ and $d_{r}$ are the number of axes in the translational and rotational workspaces.

$$
k_{r}=\left(k_{i} k_{j} k_{k}\right)^{d_{r} / d_{t}}
$$

Then $N$ (the number of $\theta_{1}$ samples) is chosen so that $K_{r}$ from (16) is as close as possible to $k_{r}$ from (15) where $\bar{\theta}_{1}$ is the maximum tip angle defining the angular workspace boundary and the floor function truncates the decimal portion of the expression since only integer numbers of samples are possible. For example, it can be found from (16) that for $\bar{\theta}_{1}=45^{\circ}, K_{r}$ can be chosen from $\{6,8,34,58, \ldots\}$ for $N=\{1,2,3,4, \ldots\}$.

$$
K_{r}=1+\sum_{n=1}^{N} \text { floor }\left(\frac{2 \pi N}{\bar{\theta}_{1}} \sin \left(\frac{n \bar{\theta}_{1}}{N}\right)\right)
$$

\section{MANIPULATOR COMPARISON}

The design procedure is next applied to the design of an impedance type haptic interface. Only robots with low effective mass (i.e. most of their actuators in the base) are considered and design parameters are chosen to optimize static force capabilities. Three robots are considered which include the Stewart Platform, the Inoue Platform (see Figure 10) and a novel hybrid robot called the Twin-Pantograph (see Figure 11).

The Twin-Pantograph uses two 3-DOF 5-bar linkages that are actuated about their folding or waist joints $\left(q_{1}, q_{4}\right)$ to provide five degrees of freedom ( 3 translation, 2 rotation) to a cylindrical endeffector. The sixth degree of freedom (roll) is provided by a series actuator $\left(q_{7}\right)$ mounted to one of the 5-bar linkages. While many permutations of this device exist (e.g. the pantographs could be replaced by prismatic actuators, $q_{7}$ could be replaced by a leadscrew mechanism as in [11], only the version shown in Figure 11 is considered here.

The Twin-Pantograph is a 6-DOF robot with seven actuators. One waist actuator is redundant but eliminates a singularity that would occur when the platform axis lies in the plane of the five-bar linkage with a passive waist ${ }^{1}$. It can be shown that the only remaining singular configurations occur when (i) a pantograph is in a singular configuration (Figure 12a-c), (ii) the tip of a pantograph intersects its own waist axis (Figure 12c-d), (iii) a

1 Note that the same redundant actuation and sensing approach can be used with the Inoue platform. 

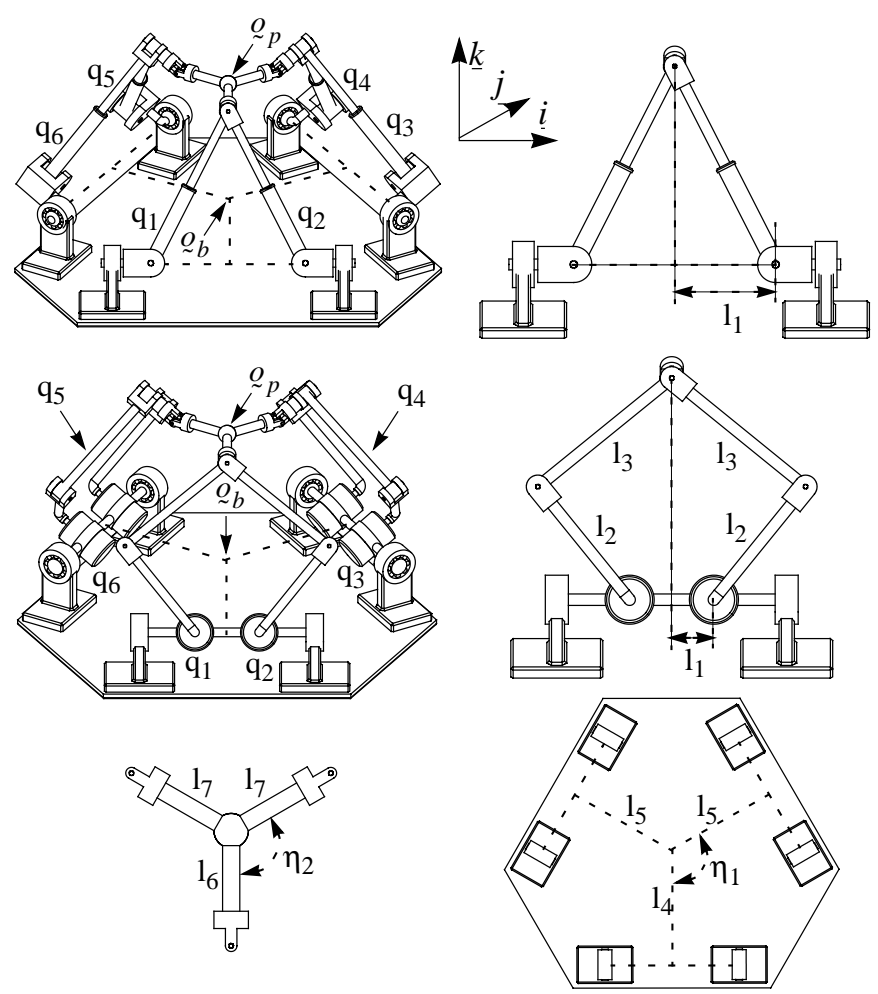

Fig. 10. Stewart \& Inoue Platform Manipulators
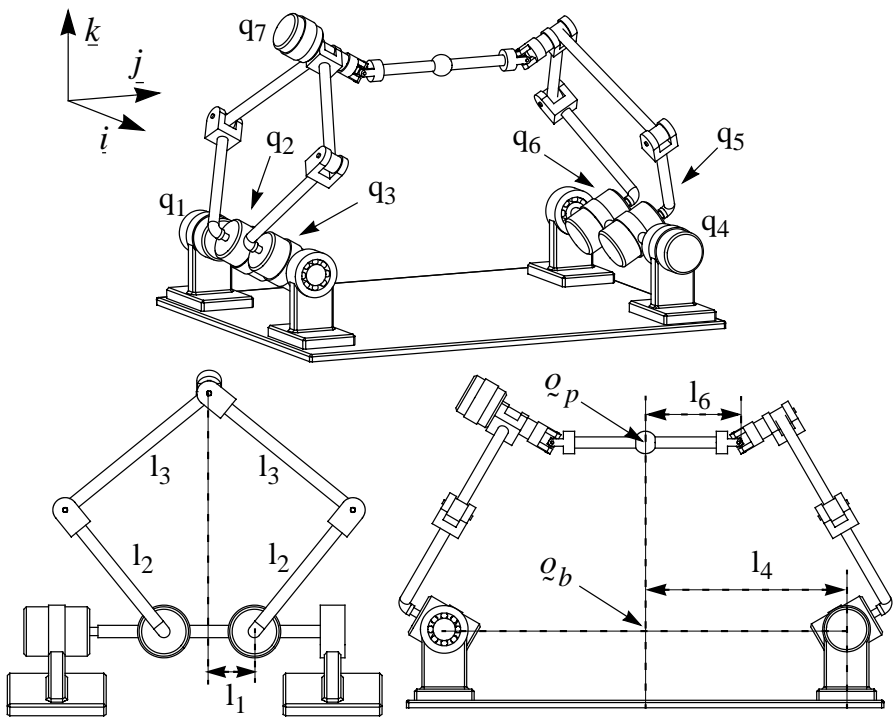

Fig. 11. Twin-Pantograph Robot

pantograph is at its workspace limit or (iv) the tips of both pantographs align with a forearm of a pantograph (i.e. a spherical wrist singularity). The pantograph singularities are, however, not particularly problematic since, by design, the singularity in Figure $15 \mathrm{a}$ is physically impossible if $l_{1}>l_{2}$, as are the singularities in Figures 15b-d if $l_{3}>\left(l_{1}+l_{2}\right)$.

Although the Twin-Pantograph uses a series actuator to roll its end-effector, the torque requirements of this actuator are small (see Table I) so its inertial contribution is not expected to exceed

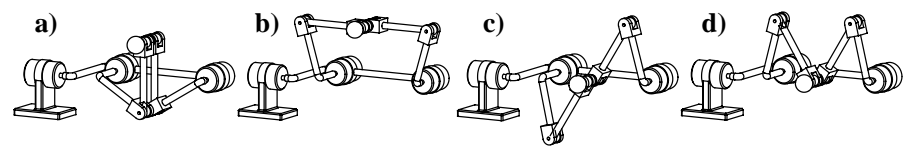

Fig. 12. Pantograph Singular Positions

that of the two additional base to platform links of the parallel platforms.

First, the semi-dextrous workspaces of the three devices are compared. A point belongs to such a semi-dextrous workspace if the platform origin $\alpha_{p}$ can be placed there and rolled, pitched and yawed $\pm 30^{\circ}$. For this comparison, the devices are given similar footprints and favourable geometries (i.e. similar forearm and upper arm lengths, singularity avoidance as described above and platforms half as large as the bases as suggested in [15]). The lengths $q$ of the prismatic actuators are allowed to range between $9 \leq q \leq 18$ and the 5-bar linkage geometries are chosen to provide a similar reach. The resulting semi-dextrous workspaces are shown in Figure 13a-c for the Stewart Platform, Inoue Platform and Twin-Pantograph respectively.
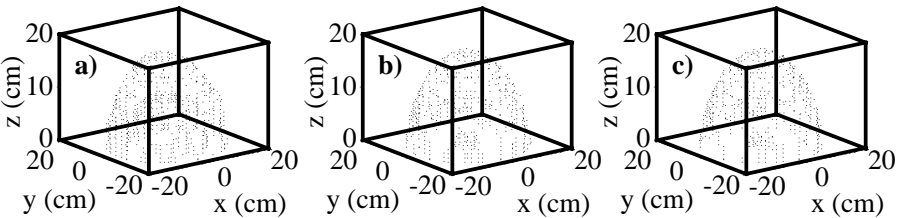

Fig. 13. Semi-Dextrous Workspace Comparison of 6-DOF Manipulators

The workspaces are all similar in size except that the Stewart Platform's has a large void in its centre due to the retraction limits of its prismatic actuators so its volume is much less than the others. If the U-joints between the 5-bar linkages and platforms of the Inoue Platform and Twin-Pantograph are constrained to $\pm 85^{\circ}$ to avoid a spherical wrist singularity, their workspaces are reduced to those shown in Figure $14 a-b$ respectively. The TwinPantograph's workspace is clearly larger.
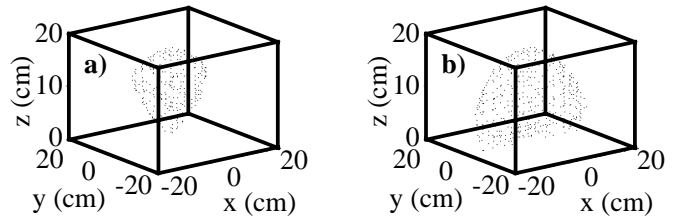

Fig. 14. Workspace Comparison with U-Joint Bend Angle Constraint

Next, static force capabilities are compared by kinematically optimizing $J^{T}(x)$ for each device. For this comparison, the TwinPantograph's redundant $\left(q_{4}\right)$ actuator is replaced by a passive joint to eliminate any unfair advantage that may result from redundant actuation so all three manipulators have square (i.e. $6 \times 6)$ Jacobian matrices.

It was suggested in [23] that a reasonable workspace size for a haptic device is $16 \mathrm{~cm}(i$ axis $) \times 10 \mathrm{~cm}(j, k$ axes $) \times \pm 45^{\circ}$. Since the parallel robots would have difficulty reaching a $\pm 45^{\circ}$ rotational workspace, it is reduced to $\pm 30^{\circ}$ for this comparison. The 
workspace centre is placed a fixed distance of $25 \mathrm{~cm}$ (measured along the $k$ axis) from the base origin $o_{b}$ (see Figures 13 and 14). This avoids a trivial result since the GII, being a kinematic isotropy index, always improves when the workspace is moved further from the base resulting in infinitely large robots. The 6DOF workspaces are discretized as shown in Table II. Since the parallel platforms are symmetric about the $j k$ plane, half of the cartesian workspace is redundant and does not affect the outcome. To eliminate unnecessary computations, the minimum position on the $i$ axis is set to 0 so the total number of discrete samples is 2,653,020. The Twin-Pantograph, on the other hand, is not symmetric because its wrist actuator operates relative to a pantograph forearm so its entire workspace is considered.

TABLE II

DISCRETE WORKSPACE

\begin{tabular}{c|c|c|c|c}
\hline \multicolumn{5}{c}{ Position } \\
\hline Axis & Min & Max & Step & Total \\
\hline $\mathrm{i}$ & $-8 \mathrm{~cm}$ & $8 \mathrm{~cm}$ & $1.0 \mathrm{~cm}$ & 17 \\
\hline $\mathrm{j}$ & $-5 \mathrm{~cm}$ & $5 \mathrm{~cm}$ & $1.0 \mathrm{~cm}$ & 11 \\
\hline $\mathrm{k}$ & $-5 \mathrm{~cm}$ & $5 \mathrm{~cm}$ & $1.0 \mathrm{~cm}$ & 11 \\
\hline
\end{tabular}

\begin{tabular}{|c|c|c|c|c|}
\hline \multicolumn{5}{|c|}{ Orientation } \\
\hline Axis & Min & Max & Step & Total \\
\hline $\mathrm{i}, \mathrm{k}$ & $-30^{\circ}$ & $30^{\circ}$ & variable & 168 \\
\hline $\mathrm{j}$ & $-30^{\circ}$ & $-30^{\circ}$ & $5^{\circ}$ & 13 \\
\hline \multicolumn{3}{|c|}{ Total discrete samples } & \multicolumn{2}{|c|}{$4,492,488$} \\
\hline
\end{tabular}

Although there are more geometric parameters than are shown in Figure 10 and Figure 11, some symmetries are introduced to keep the number of parameters manageable. Furthermore, all devices are allotted two actuator scale factors, $Q_{1}$ and $Q_{2}$. For the parallel platforms, $Q_{1}$ is applied to $q_{3}$ and $q_{6}$ and $Q_{2}$ is applied to $q_{4}$ and $q_{5}$ since the devices are symmetric about the $j k$ plane but not about the $i k$ plane. For the Twin-Pantograph, $Q_{1}$ is applied to $q_{1}$ and $Q_{2}$ is applied to $q_{7}$ since the waist $\left(q_{1}\right)$, wrist $\left(q_{7}\right)$ and shoulder $\left(q_{2}, q_{3}, q_{5}, q_{6}\right)$ joints have the most dissimilar torque demands. The resulting joint-space scaling matrices $\left(S_{J}\right)$ are shown in (17) for the Twin-Pantograph and in (18) for the parallel platforms.

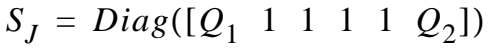

$$
\begin{aligned}
& S_{J}=\operatorname{Diag}\left(\left[\begin{array}{llllll}
1 & 1 & Q_{1} & Q_{2} & Q_{2} & Q_{1}
\end{array}\right]\right)
\end{aligned}
$$

Since a haptic interface must counteract human hand forces, the maximum force/torque capabilities reported in Table I are used to assign the $S_{T}$ matrix (19). Since these forces are relative to the participant's hand, the Jacobian is expressed in the end-effector coordinate frame prior to scaling.

$$
S_{T}=\operatorname{Diag}\left(\left[\begin{array}{llllll}
1 & 1 & 1.5 & 3.6 & 0.5 & 3.9
\end{array}\right]\right)
$$

Due to practical limits (processing speed of a SGI $\mathrm{O}^{2}$ running IRIX) the design parameter space is limited to 50 million elements. It is, therefore, possible to search a broader range or use finer sample spacing when there are fewer design parameters. Since the Twin-Pantograph has 7 free design parameters $\left(l_{1}-l_{4}\right.$, $\left.l_{6}, Q_{1}, Q_{2}\right)$, the Stewart Platform has $9\left(l_{1}, l_{4}-l_{7}, \eta_{1}, \eta_{2}, Q_{1}, Q_{2}\right)$ and the Inoue Platform has $11\left(l_{1}-l_{7}, \eta_{1}, \eta_{2}, Q_{1}, Q_{2}\right)$, search ranges and sample spacings are assigned accordingly.

The optimization problem in equation (10) is formulated using the parameter space $P$ shown in (20), where the objective function $F(p)$ is the GII described in equation (11) which uses the workspace W shown in (21), the Jacobian shown in (22), the $S_{J}$ matrix shown in (17) and (18) which contains parameters $Q_{1}$ and $Q_{2}$ from $P$ and the $S_{T}$ matrix shown in (19) which contains the human hand performance values from Table I.

$$
\begin{gathered}
P=\left\{l_{1} \ldots l_{7}, \eta_{1}, \eta_{2}, Q_{1}, Q_{2}\right\} \\
W=\left\{ \pm 8 \mathrm{~cm}(i), \pm 5 \mathrm{~cm}(j, k), \pm 30^{\circ}(i, j, k)\right\} \\
f=J^{T} \tau
\end{gathered}
$$

The culling algorithm is used to solve the optimization problem for each of the three manipulators. The discrete parameter spaces and optimal solutions are shown in Table III through Table V.

TABLE III

TWIN-PANTOGRAPH PARAMETER SPACE

\begin{tabular}{c|c|c|c|c|c}
\hline Param & Min & Max & Step & Total & Optimum \\
\hline $1_{1}$ & 0.5 & 5 & 0.5 & 10 & 3.0 \\
\hline $1_{2}$ & 10.0 & 25.0 & 1.0 & 16 & 16.0 \\
\hline $1_{3}$ & 15.0 & 30.0 & 1.0 & 16 & 22.0 \\
\hline $1_{4}$ & 1.0 & 10.0 & 1.0 & 10 & 2.0 \\
\hline $1_{6}$ & 0.5 & 6.0 & 0.5 & 12 & 3.0 \\
\hline $\mathrm{Q}_{1}$ & 0.6 & 2.0 & 0.1 & 15 & 1.9 \\
\hline $\mathrm{Q}_{2}$ & 0.005 & 0.05 & 0.005 & 10 & 0.03 \\
\hline \multicolumn{6}{c}{ Total discrete samples } \\
\hline
\end{tabular}

TABLE IV

STEWART PLATFORM PARAMETER SPACE

\begin{tabular}{c|c|c|c|c|c}
\hline Param & Min & Max & Step & Total & Optimum \\
\hline$l_{1}$ & 4.0 & 16.0 & 2.0 & 7 & 10.0 \\
\hline$l_{4}$ & 2.0 & 14.0 & 2.0 & 7 & 6.0 \\
\hline $1_{5}$ & 2.0 & 14.0 & 2.0 & 7 & 6.0 \\
\hline$l_{6}$ & 1.0 & 7.0 & 1.0 & 7 & 3.0 \\
\hline $1_{7}$ & 1.0 & 7.0 & 1.0 & 7 & 4.0 \\
\hline$\eta_{1}$ & $110^{\circ}$ & $140^{\circ}$ & $5^{\circ}$ & 7 & $120^{\circ}$ \\
\hline$\eta_{2}$ & $140^{\circ}$ & $175^{\circ}$ & $5^{\circ}$ & 8 & $170^{\circ}$ \\
\hline $\mathrm{Q}_{1}$ & 0.3 & 1.5 & 0.2 & 7 & 0.5 \\
\hline $\mathrm{Q}_{2}$ & 0.3 & 1.5 & 0.2 & 7 & 0.9 \\
\hline \multicolumn{5}{c}{ Total discrete samples } & \multicolumn{2}{c}{$46,118,408$} \\
\hline \multicolumn{6}{c}{ GII of optimum solution }
\end{tabular}


TABLE V

INOUE PLATFORM PARAMETER SPACE

\begin{tabular}{c|c|c|c|c|c}
\hline Param & Min & Max & Step & Total & Optimum \\
\hline $1_{1}$ & 1.0 & 5.0 & 1.0 & 5 & 2.0 \\
\hline $1_{2}$ & 12.0 & 22.0 & 2.0 & 6 & 16.0 \\
\hline $1_{3}$ & 20.0 & 30.0 & 2.0 & 6 & 24.0 \\
\hline $1_{4}$ & 2.0 & 8.0 & 2.0 & 4 & 2.0 \\
\hline $1_{5}$ & 2.0 & 8.0 & 2.0 & 4 & 2.0 \\
\hline $1_{6}$ & 1.0 & 5.0 & 1.0 & 5 & 2.0 \\
\hline $1_{7}$ & 1.0 & 5.0 & 1.0 & 5 & 4.0 \\
\hline$\eta_{1}$ & $100^{\circ}$ & $140^{\circ}$ & $10^{\circ}$ & 5 & $120^{\circ}$ \\
\hline$\eta_{2}$ & $130^{\circ}$ & $170^{\circ}$ & $10^{\circ}$ & 5 & $170^{\circ}$ \\
\hline $\mathrm{Q}_{1}$ & 0.5 & 1.5 & 0.25 & 5 & 0.75 \\
\hline $\mathrm{Q}_{2}$ & 0.5 & 1.5 & 0.25 & 5 & 0.75 \\
\hline \multicolumn{7}{|c}{ Total discrete samples } & & \multicolumn{2}{c}{0.215} \\
\hline
\end{tabular}

The optimum GIIs of the Twin-Pantograph, Stewart Platform, and Inoue Platform are 0.327, 0.096 and 0.215 respectively. The static force GII of the Twin-Pantograph is 3.4 and 1.5 times larger than the GIIs of the parallel platforms. Therefore, the TwinPantograph is much better suited to the demands of a haptic interface since it can make more efficient use of its actuators than the parallel platforms can.

\section{DESIGN OF A HAPTIC PEN}

With its large semi-dextrous workspace and favourable static forces capabilities, the Twin-Pantograph is the most viable candidate for the design of a haptic pen. As predicted, the serial wrist actuator should be very small (i.e. $1 / 63$ the torque of the waist actuator) according to Table III. A light-weight actuator is under development for this purpose but in the meantime, this joint is made passive. This simplified device ( 5 active DOF, 1 passive DOF) lends itself well to applications which do not rely on reaction torques from axial rotations.

To fully exploit the device's motion range, the rotational workspace is extended to $\pm 45^{\circ}$ and to further enhance its static force capabilities, the redundant waist actuator $\left(q_{4}\right)$ is reintroduced. With only 5 active degrees-of-freedom and a redundant actuator, the Twin-Pantograph is symmetric about both the $j k$ plane and the $i k$ plane so only one quarter of its translational workspace needs to be considered to rigorously evaluate its performance. This allows the workspace to be discretized more finely as shown in Table VI.

Since a singularity is eliminated by introducing the redundant actuator, the GII improves when the base length $\left(l_{4}\right)$ is increased since this increases the distance between the workspace and the actuators. Therefore, $l_{4}$ is fixed at $8.0 \mathrm{~cm}$ to keep the height of the device reasonable while providing adequate clearance for the shoulder actuators. The resulting device has 5 free design
TABLE VI

5-DOF DISCRETE WORKSPACE

\begin{tabular}{|c|c|c|c|c|}
\hline \multicolumn{5}{|c|}{ Position } \\
\hline Axis & Min. & Max. & Step & Total \\
\hline $\mathrm{i}$ & $0 \mathrm{~cm}$ & $8 \mathrm{~cm}$ & $0.5 \mathrm{~cm}$ & 17 \\
\hline $\mathrm{j}$ & $0 \mathrm{~cm}$ & $5 \mathrm{~cm}$ & $0.5 \mathrm{~cm}$ & 11 \\
\hline $\mathrm{k}$ & $-5 \mathrm{~cm}$ & $5 \mathrm{~cm}$ & $0.5 \mathrm{~cm}$ & 21 \\
\hline \multicolumn{5}{|c|}{ Orientation } \\
\hline Axis & Min. & Max. & Step & Total \\
\hline $\mathrm{i}, \mathrm{k}$ & $-45^{\circ}$ & $45^{\circ}$ & $\mathrm{n} / \mathrm{a}$ & 617 \\
\hline
\end{tabular}

parameters which are shown with the optimal solution in Table VII. Note that $l_{l}$ is assigned a minimum value of $2.4 \mathrm{~cm}$ to account for the width of the shoulder actuators and $l_{6}$ is assigned a minimum length of $7.0 \mathrm{~cm}$ so that the end-effector can be held comfortably by a human hand.

TABLE VII

5-DOF TWIN-PANTOGRAPH PARAMETER SPACE

\begin{tabular}{c|c|c|c|c|c}
\hline Dim. & Min. & Max. & Step & Total & Optimum \\
\hline $\mathrm{l}_{1}$ & 2.4 & 7.0 & 0.2 & 24 & 2.4 \\
\hline $\mathrm{l}_{2}$ & 10.0 & 30.0 & 0.5 & 41 & 17.0 \\
\hline $\mathrm{l}_{3}$ & 15.0 & 35.0 & 0.5 & 41 & 23.0 \\
\hline $\mathrm{l}_{6}$ & 7.0 & 11.0 & 0.2 & 21 & 7.0 \\
\hline $\mathrm{Q}_{1}$ & 0.5 & 3.0 & 0.1 & 26 & 1.6 \\
\hline \multicolumn{3}{c|}{ Total discrete samples } & \multicolumn{2}{c}{$22,027,824$} \\
\hline \multicolumn{3}{c}{ GI of optimum solution } & \multicolumn{3}{c}{0.234} \\
\hline
\end{tabular}

The workspace position (i.e. $25 \mathrm{~cm}$ away from the base) was chosen somewhat arbitrarily. Increasing this distance is known to improve the GII but also increases the mass and footprint of the device. To see how performance is affected by workspace positioning, the device is re-optimized for workspace distances ranging from $15 \mathrm{~cm}$ to $40 \mathrm{~cm}$. The results are shown in Table VIII.

TABLE VIII

EFFECT OF WORKSPACE DISTANCE ON GII

\begin{tabular}{c|c|c|c|c|c|c}
\hline Distance & $\mathrm{l}_{1}$ & $\mathrm{l}_{2}$ & $\mathrm{l}_{3}$ & $\mathrm{l}_{6}$ & $\mathrm{Q}_{1}$ & GII \\
\hline $15 \mathrm{~cm}$ & 2.4 & 13.0 & 16.0 & 7.0 & 1.6 & 0.089 \\
\hline $20 \mathrm{~cm}$ & 2.4 & 14.5 & 20.0 & 7.0 & 1.7 & 0.182 \\
\hline $25 \mathrm{~cm}$ & 2.4 & 17.0 & 23.0 & 7.0 & 1.6 & 0.234 \\
\hline $30 \mathrm{~cm}$ & 3.6 & 19.5 & 27.0 & 7.0 & 1.6 & 0.262 \\
\hline $35 \mathrm{~cm}$ & 3.6 & 22.5 & 30.5 & 7.0 & 1.6 & 0.280 \\
\hline $40 \mathrm{~cm}$ & 4.0 & 25.5 & 34.5 & 7.0 & 1.6 & 0.292 \\
\hline
\end{tabular}

The position/velocity sensing capabilities are considered next by selecting optimum sensor resolutions for each of the designs in Table VIII. This is accomplished by re-formulating the optimization problem using the Jacobian in (23) and the parameter space $P$ in (24) which contains no geometric quantities $\left(l_{1}\right.$ through $l_{6}$ are taken from Table VIII) but only the design 
parameter $Q_{1}$ which is the ratio between waist and should joint sensor resolution $\left(R_{w} / R_{s}\right)$. In accordance with the force/torque optimization, the workspace $\mathrm{W}$ in (25) is used.

$$
\begin{gathered}
\dot{q}=J(x) \dot{x} \\
P=\left\{Q_{1}\right\} \\
W=\left\{ \pm 8 c m(i), \pm 5 c m(j, k), \pm 45^{\circ}(i, j, k)\right\}
\end{gathered}
$$

It is assumed that to maintain a given velocity signal quality, the required position sensor resolution is inversely proportional to the maximum joint velocity. Therefore, the $S_{J}$ matrix in (26) is used to find the optimal position sensor resolution ratio between the shoulder and waist joints $\left(R_{w} / R_{s}\right)$ and the $S_{T}$ matrix is assigned to the maximum velocity capabilities of the human hand as in (27). From Figure 8, a human hand can produce a maximum linear velocity of $500 \mathrm{~cm} / \mathrm{s}$ inside a $16 \mathrm{~cm}$ workspace and maximum angular velocity of $30 \mathrm{rad} / \mathrm{s}$ inside a $\pm 45^{\circ}$ workspace. Since the participants in the velocity experiments were allowed to choose the direction that maximized their performance, these values are applied to all directions equally.

$$
\begin{aligned}
& S_{J}=\operatorname{Diag}\left(1 / R_{s}\left[\begin{array}{llllll}
R_{s} & R_{w} & R_{w} & R_{s} & R_{w} & R_{w}
\end{array}\right]\right) \\
& =\operatorname{Diag}\left(\left[\begin{array}{llllll}
1 & Q_{1} & Q_{1} & 1 & Q_{1} & Q_{1}
\end{array}\right]\right) \\
& S_{T}=\operatorname{Diag}\left(1 / v_{i}\left[\begin{array}{lllll}
v_{i} & v_{j} & v_{k} & \omega_{i} & \omega_{k}
\end{array}\right]\right) \\
& =\operatorname{Diag}\left(\left[\begin{array}{lllll}
1 & 1 & 1 & 0.06 & 0.06
\end{array}\right]\right)
\end{aligned}
$$

The optimization is carried out for each of the workspace positions in Table VIII to obtain the optimal encoder resolutions and velocity GIIs in Table IX. The GII from the force/torque optimization is plotted using a solid line and the GII from the position sensor optimization is plotted using a dashed line in Figure 15.

TABLE IX

OPTIMAL POSITION SENSOR RESOLUTION RATIOS

\begin{tabular}{c|c|c|c|c|c|c|c}
\hline & $10 \mathrm{~cm}$ & $15 \mathrm{~cm}$ & $20 \mathrm{~cm}$ & $25 \mathrm{~cm}$ & $30 \mathrm{~cm}$ & $35 \mathrm{~cm}$ & $40 \mathrm{~cm}$ \\
\hline$R_{W} / R_{s}$ & $\mathrm{n} / \mathrm{a}$ & 1.63 & 1.67 & 1.63 & 1.64 & 1.62 & 1.59 \\
\hline$G I I$ & 0.0 & 0.105 & 0.186 & 0.232 & 0.269 & 0.285 & 0.303 \\
\hline
\end{tabular}

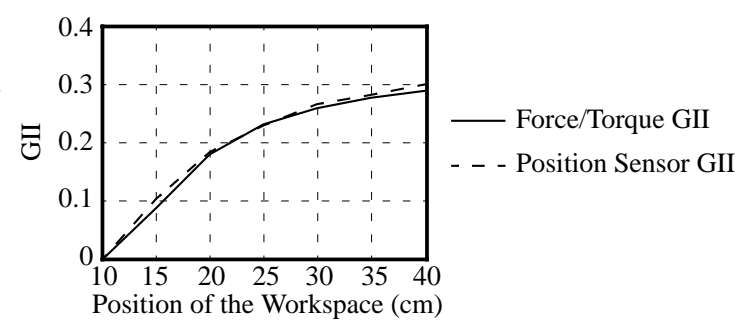

Fig. 15. Plot of GII vs. Workspace Position

Figure 15 shows nearly identical GIIs for both force/torque and position sensing at each workspace position. This is a fortunate result that simplifies the task of choosing a workspace position since there is no trade-off to make between force/torque and position sensing performance. They both improve as the workspace is moved further from the base, with rapid deterioration below $20 \mathrm{~cm}$ and marginal improvement above $25 \mathrm{~cm}$. Therefore, a workspace position between $20-25 \mathrm{~cm}$ provides the best trade-off between performance and compactness. Note that regardless of workspace position, the waist actuators should have approximately 1.6 time the torque and 1.6 times the sensor resolution of the shoulder actuators.

A prototype Twin-Pantograph haptic pen was built that has its workspace $20 \mathrm{~cm}$ from the base (see Figure 16). Mass was minimized by using hollow, magnesium clevises, carbon fibre links and low inertia, rare-earth magnet Maxon motors. Steel weights were mounted behind the shoulder motors to achieve static balance at a location just outside the workspace boundary that is nearest to the motors. Remaining gravitational effects are small and can be compensated by active control. Backlash and friction were minimized by using direct-drive motors and roller bearings in all of the passive joints except for the universal joints which are made from Delrin. Since all motors are direct drive, larger actuators were chosen for the waist joints to satisfy the additional torque requirements. The position of each active joint is sensed using a 4000cpt optical encoder. Although the sensor resolution could be higher at the waist joints, this was too costly to implement on the prototype.

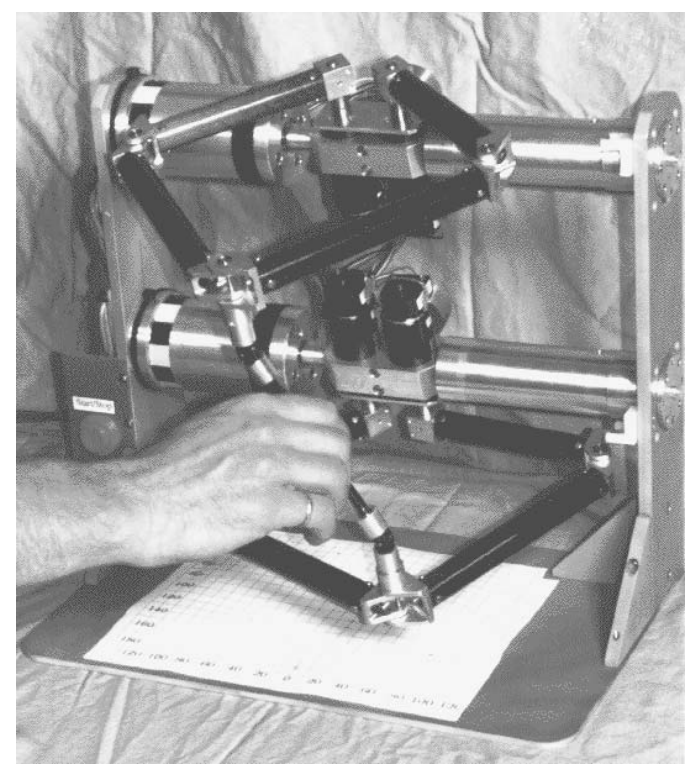

Fig. 16. The Twin-Pantograph Haptic Pen

A mass matrix $D(28)$ is computed numerically for the TwinPantograph haptic pen at its home position (i.e. $x, y=0, z=20 \mathrm{~cm}$, zero rotation) using Pro/MECHANICA ${ }^{\mathrm{TM}}$. Its value is shown in (29). 


$$
\begin{gathered}
{\left[\begin{array}{c}
f(N) \\
\tau(N c m)
\end{array}\right]=D\left[\begin{array}{c}
\ddot{x}\left(\mathrm{~cm} / \mathrm{s}^{2}\right) \\
\dot{\omega}\left(\mathrm{rad} / \mathrm{s}^{2}\right)
\end{array}\right]} \\
D=\left[\begin{array}{ccccc}
190 & 0.197 & -0.179 & 0.333 & 1.52 \\
0.197 & 226 & -0.035 & -346 & -0.515 \\
-0.179 & -0.035 & 156 & -0.642 & -0.335 \\
0.333 & -346 & -0.642 & 10300 & 12 \\
1.52 & -0.515 & -0.335 & 12 & 12600
\end{array}\right] \times 10^{-5}
\end{gathered}
$$

The robot specifications listed in Table $\mathrm{X}$ are either measured directly or are derived from the mass and Jacobian matrices and the actuator and sensor specifications. All values correspond to the robot at its home position except for weight which is the gravitational force felt by the hand while holding the end-effector in an upright position and is presented as a workspace inclusive range. The stiffness and damping coefficients are obtained using a tuned PD controller at a control rate of $1 \mathrm{KHz}$ with the end-point velocity computed from low-pass filtered finite difference position readings. The minimum force is the force required to overcome static friction and the dynamic range is the ratio between the minimum and peak force/torque capabilities.

TABLE $X$

HAPTIC PEN PERFORMANCE SPECIFICATIONS

\begin{tabular}{c|c|c|c|c|c}
\cline { 2 - 6 } & \multicolumn{3}{c|}{ Translation Axis } & \multicolumn{2}{c}{ Rotation Axis } \\
\cline { 2 - 6 } & $\mathrm{x}$ & $\mathrm{y}$ & $\mathrm{z}$ & $\mathrm{x}$ & $\mathrm{z}$ \\
\hline Workspace & $\pm 6 \mathrm{~cm}$ & $\pm 3.75 \mathrm{~cm}$ & $\pm 3.75 \mathrm{~cm}$ & $\pm 45^{\circ}$ & $\pm 45^{\circ}$ \\
\hline Spatial Res & $142 \mu \mathrm{m}$ & $314 \mu \mathrm{m}$ & $175 \mu \mathrm{m}$ & $0.122^{\circ}$ & $0.099^{\circ}$ \\
\hline Min F/T & $0.022 \mathrm{~N}$ & $0.045 \mathrm{~N}$ & $0.023 \mathrm{~N}$ & $0.19 \mathrm{Ncm}$ & $0.18 \mathrm{Ncm}$ \\
\hline Cont F/T & $5.0 \mathrm{~N}$ & $3.3 \mathrm{~N}$ & $4.1 \mathrm{~N}$ & $34 \mathrm{Ncm}$ & $41 \mathrm{Ncm}$ \\
\hline Peak F/T & $48 \mathrm{~N}$ & $21 \mathrm{~N}$ & $40 \mathrm{~N}$ & $324 \mathrm{Ncm}$ & $396 \mathrm{Ncm}$ \\
\hline Dyn Range & $2200: 1$ & $470: 1$ & $1700: 1$ & $1700: 1$ & $2200: 1$ \\
\hline Eff Mass & $190 \mathrm{~g}$ & $226 \mathrm{~g}$ & $156 \mathrm{~g}$ & $10300 \mathrm{gcm}{ }^{2}$ & $12600 \mathrm{gcm}{ }^{2}$ \\
\hline Peak Accel & $25.8 \mathrm{G}$ & $9.5 \mathrm{G}$ & $26.1 \mathrm{G}$ & $3147 \mathrm{~s}^{-2}$ & $3143 \mathrm{~s}^{-2}$ \\
\hline Max Stiff & $16 \mathrm{~N} / \mathrm{cm}$ & $12 \mathrm{~N} / \mathrm{cm}$ & $13 \mathrm{~N} / \mathrm{cm}$ & $874 \mathrm{Ncm}$ & $1076 \mathrm{Ncm}$ \\
\hline Max Damp & $0.44 \mathrm{Ns} / \mathrm{cm}$ & $0.5 \mathrm{Ns} / \mathrm{cm}$ & $0.36 \mathrm{Ns} / \mathrm{cm}$ & $24 \mathrm{Nscm}$ & $30 \mathrm{Nscm}$ \\
\hline Weight & \multicolumn{5}{c}{$70 \mathrm{~g}-130 \mathrm{~g}$} \\
\hline
\end{tabular}

The Twin-Pantograph's rotational workspace is extremely large for a robot which has all of its motors in the base. It has low unbalanced weight (e.g. by comparison a standard ball-point pen weighs about $15 \mathrm{~g}$ ) so little active gravity compensation is required. Its effective mass is also low making the device comfortable to use and providing high acceleration capabilities for good stiff surface emulation. It has high static force capabilities and adequate position resolution for reasonable control stiffness and damping gains using inexpensive sensors.

The haptic pen has been used to simulate two dissimilar virtual environments. One is a virtual pencil that can write on the surface of a stiff bounding box. It contains a compliant button which can be pressed from the top until a positive click is felt which erases all pencil marks from the bounding box. The other is a virtual excavator that can dig into its surrounding landscape. The motion range of the Twin-Pantograph is constrained to respect the joint limits of the virtual excavator and displays the ground interaction forces described in [7] during digging. The graphical environments for these two simulations are shown in Figure 17.
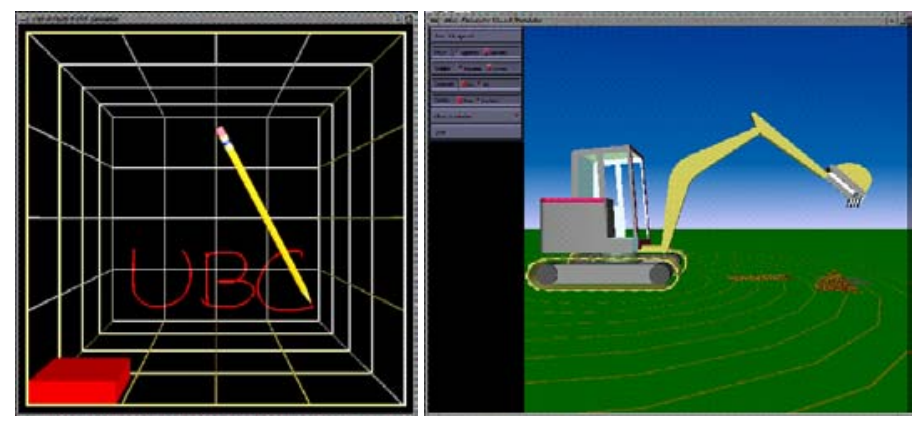

Fig. 17. Graphical Environments

The haptic pen is controlled using a PD+B controller where $\mathrm{B}$ is a braking pulse [22] that is applied upon contact with a stiff environment and is proportional to end-point velocity. The desired force and torque at the centre of the haptic pen is converted into a desired force for each pantograph (i.e. each end of the haptic pen) before computing joint torques. This control strategy is shown in Figure 18 where $J_{T}^{-T}$ is the Jacobian matrix for the top pantograph and $J_{B}{ }^{-T}$ is the Jacobian matrix for the bottom pantograph.

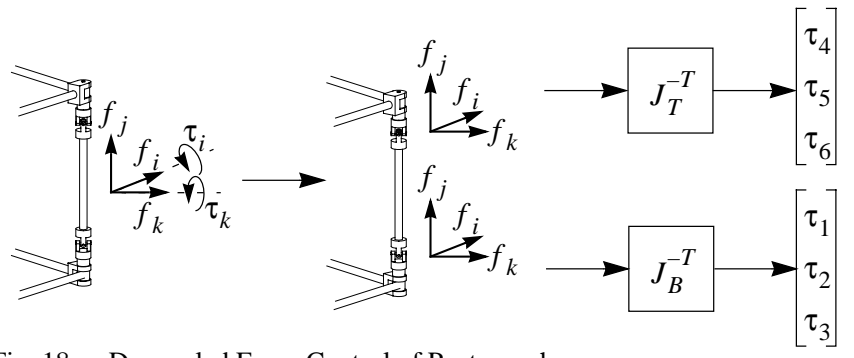

Fig. 18. Decoupled Force Control of Pantographs

Although many optimization strategies exist for allocating torques among redundant actuators, controlling the device as two 3 -DOF force sources has a number of advantages. Internal forces are avoided and it is much simpler to compute two $3 \times 3$ inverse Jacobians (i.e. one for each pantograph) than it is to optimize one $6 \times 5$ pseudo-inverse Jacobian for the purposes of control. The computational savings allow for a higher sampling rate which increases the stability margin. This approach also improves the realism of simulations with vector-like slaves such as the virtual pencil since the forces acting on the two vector end-points are decoupled in the controller. For example, the eraser end of the pencil feels completely free while the tip is being pressed firmly against an obstacle and vise versa. 
Stability and robustness were examined by recording motor currents and penetration depths while one end of the haptic pencil was pressed against each surface and intersection of the stiff virtual box. The stiff box was implemented using proportional gains of $3 \mathrm{~N} / \mathrm{cm}$ (horizontal) and $2.1 \mathrm{~N} / \mathrm{cm}$ (vertical), differential gains of $0.05 \mathrm{Ns} / \mathrm{cm}$ (horizontal) and $0.02 \mathrm{Ns} / \mathrm{cm}$ (vertical), and braking gains of $6 \mathrm{Ns} / \mathrm{cm}$ (horizontal) and $3 \mathrm{Ns} / \mathrm{cm}$ (vertical) at the contacting end of the haptic pencil. The top three traces in Figure 19 are the control signals applied to the three lower actuators $q_{1}$ through $q_{3}$ and the bottom three traces are the penetration depths of the pencil tip into the virtual walls and floor. The pencil is lying flat on the floor without any human contact between $t_{0}$ and $t_{1}$, is held in free space between $t_{1}$ and $t_{2}$, is pressed into the walls, floor and adjoining corners between $t_{2}$ and $t_{3}$, is pressed into the corner adjoining all three surfaces between $t_{3}$ and $t_{4}$ and is returned to free space between $t_{4}$ and $t_{5}$. Note the correspondence between penetration depths and motor currents and the absence of any extraneous oscillations. In Figure 20, the trajectory of the pencil tip with respect to the boundary of the stiff box is shown on the left and the corresponding reaction forces are shown on the right. The trajectory is free from oscillations during surface penetrations and reaction forces never exceeded $1.0 \mathrm{~N}$.

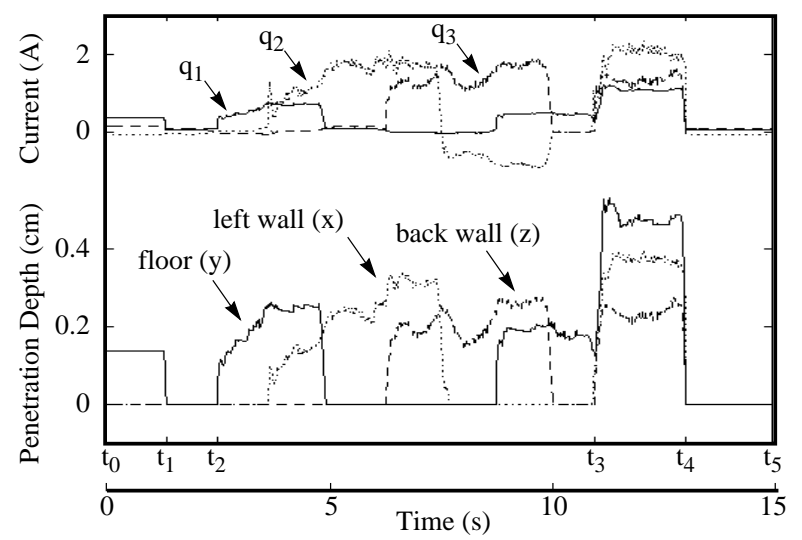

Fig. 19. Motor Currents \& Wall Penetration Depths
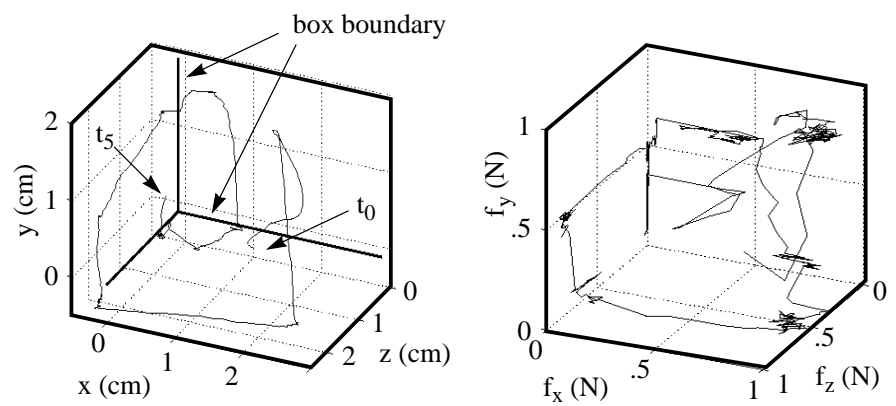

Fig. 20. Tip Position \& Reaction Force

\section{CONCLUSIONS}

To mimic any passive environment that a human hand can distinguish, a haptic interface must simulate a broad range of impedances. It was reasoned here that the impedance range of a device is widened by minimizing physical impedance and by matching static force and position sensing capabilities to those of the human hand.

It was proposed here that these attributes could be obtained by using a robot with most of its actuators in the base, by using components with low mass and friction, and by optimizing static force capabilities through kinematic design. A design procedure that was previously proposed by the authors was used for kinematic optimization. It was used to find the geometry and actuators that would best satisfy the static force criteria obtained in a biomechanics experiment that investigated achievable human hand force/torque. The process was repeated for three 6-DOF devices including the Stewart platform, the Inoue platform and a novel hybrid device called the Twin-Pantograph. Since the TwinPantograph produced the most suitable static forces and had the largest semi-dextrous workspace, it was selected as the basis for the experimental haptic pen. Optimal sensor resolutions were chosen for the Twin-Pantograph by using data from a second biomechanics experiment that investigated achievable human hand velocity.

An over-actuated version of the Twin-Pantograph with passive roll was designed, built and controlled to simulate two virtual environments including a virtual pencil and a virtual excavator. Some performance characteristics were measured and the qualitative performance of the device was examined in a number of applications with excellent results.

\section{ACKNOWLEDGMENT}

The authors would like to thank Simon Bachmann and Simon DiMaio for their valuable contributions.

This work is supported by the Canadian IRIS Network of Centres of Excellence, a scholarship from the Natural Sciences and Engineering Research Council of Canada (NSERC) and the J.K. Zee memorial fellowship.

\section{REFERENCES}

[1] S.D. Adams, P.J. Peterson, "Maximum Voluntary Hand Grip Torque for Circular Electrical Connectors", Human Factors, v. 30, no. 6, pp. 733-745, 1988.

[2] P.A. Anderson, C.E. Chanoski, D.L. Devan, B.L. McMahon, E.P. Whelan, "Normative Study of Grip and Wrist Flexion Strength Employing a BTE Work Simulator", The Journal of Hand Surgery, v. 15A, no. 3, pp. 420-425, May 1990.

[3] T.L. Brooks, "Telerobotic Response Requirements", Proc. IEEE Int. Conf. Sys. Man Cyb. (Los Angeles, California), pp. 113-120, Nov. 4-7, 1990.

[4] K. Cleary, T. Brooks, "Kinematic Analysis of a Novel 6-DOF Parallel Manipulator”, Proc. IEEE Int. Conf. Robotics \& Auto. (Atlanta, USA), pp. 
708-713, May 2-6, 1993.

[5] J.E. Colgate, G.G. Schenkel, "Passivity of a Class of Sampled-Data Systems: Application to Haptic Interfaces", J. Robotic Sys., v. 14, no. 1, pp. 37-47, Jan. 1997.

[6] C.L. Collins, G.L. Long, "The Singularity Analysis of an In-Parallel Hand Controller for Force-Reflected Teleoperation", IEEE Trans. Robotics \& Auto., v. 11, no. 5, pp. 661-669. Oct. 1995.

[7] S.P. DiMaio, S.E. Salcudean, C. Reboulet, S. Tafazoli, K. Hashtrudi-Zaad, "A Virtual Excavator for Controller Development and Evaluation", Proc. IEEE Int. Conf. Robotics \& Auto. (Leuven, Belgium), pp. 52-58, May 16$23,1998$.

[8] E.F. Fichter, "A Stewart Platform-Based Manipulator: General Theory and Practical Construction”, Int. J. Robotics Res., v. 5, no. 2, pp. 157-182, 1986.

[9] V. Hayward, J. Choksi, G. Lanvin, C. Ramstein, "Design and MultiObjective Optimization of a Linkage for a Haptic Interface", Proc. ARK '94, $4^{\text {th }}$ Int. Workshop on Adv. in Robot Kin. (Ljubliana, Slovenia), pp. 352359, June 1994.

[10] H. Iwata, "Artificial Reality with Force-Feedback: Development of Desktop Virtual Space with Compact Master Manipulator", SIGGRAPH (Dallas, Texas), v. 24, no. 4, pp. 165-170, Aug. 6-10, 1990.

[11] H. Iwata, "Pen-based Haptic Virtual Environment", IEEE Int. Symp. Virtual Reality (Seattle, Washington), pp. 287-292, 1993.

[12] A.J. Kelley, S.E. Salcudean, “The Development of a Force-Feedback Mouse and its Integration into a Graphical User Interface”, Proc. Int. Mech. Eng. Cong. \& Expo. (Chicago, Illinois), DSC-Vol. 55-1, pp. 287-294, Nov. 6-11, 1994.

[13] T. Kotoku, K. Komoriya, K. Tanie, “A Force Display System for Virtual Environments and its Evaluation", IEEE Int. Workshop on Robot \& Human Comm. (Tokyo, Japan), pp. 246-251, Sept. 1-3, 1992.

[14] R. Kurtz, V. Hayward, "Multiple-Goal Kinematic Optimization of a Parallel Spherical Mechanism with Actuator Redundancy", IEEE Trans. Robotics \& Auto., v. 8, no. 5, pp. 644-651, Oct. 1992.

[15] D.A. Lawrence, J.D. Chapel, "Performance Trade-Offs for Hand Controller Design”, Proc. IEEE. Int. Conf. Robotics \& Auto. (SanDiego, California), pp. 3211-3216, May 1994.

[16] C.D. Lee, D.A. Lawrence, L.Y. Pao, "Guaranteed Convergence Rates for Five Degree of Freedom In-Parallel Haptic Interface Kinematics", Proc. IEEE Int. Conf. Robotics \& Auto. (Detroit, Michigan), pp. 3267-3274, May 10-15, 1999.

[17] S. Leguay-Durand, C. Reboulet, "Optimal Design of a Redundant Spherical Parallel Manipulator”, Robotica, Int. J. Info., Edu. \& Res. in Robotics \& Art. Intell., v. 15, pp. 399-405, part 4, Jul.-Aug. 1997.

[18] G.L. Long, C.L. Collins, "A Pantograph Linkage Parallel Platform Master Hand Controller for Force-Reflection”, Proc. IEEE Int. Conf. Robotics \& Auto. (San Diego, California), v. 4, pp. 3211-3216, May 9-12, 1994.

[19] O. Ma, J. Angeles, "Optimum Design of Manipulators Under Dynamic Isotropy Conditions", Proc. IEEE Int. Conf. Robotics \& Auto. (Atlanta, Georgia), pp. 470-475, (May 2-6, 1993).

[20] T.H. Massie, J.K. Salisbury, "The PHANToM Haptic Interface: A Device for Probing Virtual Objects", Proc. Int. Mech. Eng. Congress \& Exposition (Chicago, USA), Vol. DSC-Vol. 55-1, pp. 295-301, Nov. 6-11, 1994.

[21] P.A. Millman, M. Stanley, J.E. Colgate, "Design of a Four Degree-ofFreedom Force-Reflecting Manipulandum with a Specified Force/Torque Workspace", Proc. IEEE Int. Conf. Robotics \& Auto. (Sacramento, California), pp. 1488-1493, Apr. 9-11, 1991.

[22] S.E. Salcudean, T.D. Vlaar, "On the Emulation of Stiff Walls and Static Friction With a Magnetically Levitated Input/Output Device", Trans. ASME - J. Dyn. Sys. Meas. \& Cont., v. 119, no. 1, pp. 127-132, Mar. 1997.

[23] L. Stocco, S.E. Salcudean, "A Coarse-Fine Approach to Force-Reflecting Hand Controller Design”, Proc. IEEE Int. Conf. Robotics \& Auto. (Minneapolis, Minnesota), v. 1, pp. 404-410, Apr. 22-28, 1996.

[24] L. Stocco, S.E. Salcudean, F. Sassani, "Fast Constrained Global Minimax
Optimization of Robot Parameters", Robotica, Int. J. Info., Edu. \& Res. in Robotics \& Art. Intell., v. 16, pp. 595-605, 1998.

[25] L.J. Stocco, S.E. Salcudean, F. Sassani, "On the Use of Scaling Matrices for Task-Specific Robot Design”, to appear IEEE Trans. Robotics \& Auto., 1999.

[26] J. Vertut, "Control of Master Slave Manipulators and Force Feedback", Proc. 1977 Joint Auto. Control Conf., 1977.

[27] W.E. Woodson, "Human Factors Design Handbook", McGraw Hill, 1981.

[28] H. Yokoi, J. Yamashita, Y. Fukui, M. Shimojo, “Development of 3D-Input Device for Virtual Surface Manipulation", $3{ }^{\text {rd }}$ IEEE Int. Workshop on Robot \& Human Commun. (Nagoya, Japan), pp. 134-139, July 18-20, 1994.

[29] K.E. Zanganeh, J. Angeles, "Kinematic Isotropy and the Optimum Design of Parallel Manipulators”, Int. J. Robotics Res., v. 16, no. 2, pp.185-197, Apr. 1997. 